\title{
Genome-wide association study of prolactin levels in blood plasma and cerebrospinal fluid
}

\author{
Lyndsay A. Staley ${ }^{1 \dagger}$, Mark T. W. Ebbert ${ }^{1 \dagger}$, Sheradyn Parker ${ }^{1}$, Matthew Bailey ${ }^{2}$, for the Alzheimer's Disease
} Neuroimaging Initiative, Perry G. Ridge', Alison M. Goate ${ }^{3}$ and John S. K. Kauwe ${ }^{1^{*}}$

From 12th Annual Biotechnology and Bioinformatics Symposium (BIOT-2015)

Provo, UT, USA. 10-11 December 2015

\begin{abstract}
Background: Prolactin is a polypeptide hormone secreted by the anterior pituitary gland that plays an essential role in lactation, tissue growth, and suppressing apoptosis to increase cell survival. Prolactin serves as a key player in many life-critical processes, including immune system and reproduction. Prolactin is also found in multiple fluids throughout the body, including plasma and cerebrospinal fluid (CSF).

Methods: In this study, we measured prolactin levels in both plasma and CSF, and performed a genome-wide association study. We then performed meta-analyses using METAL with a significance threshold of $p<5 \times 10^{-8}$ and removed SNPs where the direction of the effect was different between the two datasets.

Results: We identified 12 SNPs associated with increased prolactin levels in both biological fluids.

Conclusions: Our efforts will help researchers understand how prolactin is regulated in both CSF and plasma, which could be beneficial in research for the immune system and reproduction.
\end{abstract}

Keywords: Association, Genetics, CSF, Plasma, Prolactin

\section{Background}

Prolactin, a hormone mostly secreted from the lactotroph cells within the anterior pituitary gland [1] and expressed by the $P R L$ gene, plays an important role in milk lactation for pregnant women [1], helps regulate the menstrual cycle, and also affects reproduction, metabolism, homeostasis, tissue growth, osmoregulation, immunoregulation, and behavior [2, 3]. Prolactin levels are regulated in a short-loop feedback mechanism by prolactin inhibitory factors (PIF), dopamine being an important example [4]. This feedback system changes during pregnancy, and prolactinomas, hypothyroidism, medications, stress, exercise, herbs, and certain foods can also affect prolactin levels $[5,6]$. Prolactin has also

\footnotetext{
*Correspondence: Kauwe@byu.edu

${ }^{\dagger}$ Equal contributors

'Department of Biology, Brigham Young University, Provo, UT 84602, USA

Full list of author information is available at the end of the article
}

been shown to suppress apoptosis, and increase survival and function of cells, including T-lymphocytes [7].

Cerebrospinal fluid (CSF) and plasma separated by the blood-brain barrier and levels of expression in these biological fluids are often independent, suggesting the genes are regulated independently across tissues on either side of the blood-brain barrier [8]. Currently, little is known about genetic markers that affect prolactin expression in plasma or CSF. In this study we conducted a genome-wide association study of prolactin levels in the CSF and in the plasma of individuals from two datasets, looking for SNPs that are associated with prolactin levels in both CSF and plasma. Further research of the variants we identified will help researchers further understand how prolactin is regulated across multiple tissues in the human body and how it affects human health. 


\section{Methods}

\section{Subjects and data description}

CSF and plasma samples were collected from the Knight-Alzheimer's Disease Research Center at Washington University School of Medicine (Knight ADRC) and from the Alzheimer's Disease Neuroimaging Initiative (ADNI). In this study, we used 297 CSF and 347 plasma samples from ADNI, and 246 CSF and 240 plasma samples from Knight ADRC. The majority of the samples were controls, although $7 \%$ of Knight ADRC samples were Alzheimer's disease cases, and $15 \%$ of ADNI samples were AD cases. Levels for 190 biomarkers were measured for each sample using the Human DiscoveryMAP Panel v1.0 and a Luminex 100 platform [9] and the samples were genotyped using the Illumina 610 or the Omniexpress chip. A description of the collection methods and the Knight ADRC samples has been previously published $[10,11]$ and the ADNI samples were collected as part of the ADNI biomarker study [12], and were obtained from the ADNI database (adni.loni.usc.edu). All samples were of European descent, and varied in age from 58 to 91 years, with an average age of 76 years, for the ADNI samples, and varied in age from 49 to 91 years, with an average age of 73 years, for the Knight ADRC samples. All individuals whose data were included in this study were explicitly consented, following appropriate Institutional Review Board policies.

\section{SNP imputation}

SNPs were imputed as previously described [13]. Beagle was used to impute SNPs from the data from the 1000 Genomes Project (June 2012 release). Imputed SNPs with the following criteria were removed: (1) an $\mathrm{r}^{2}$ of 0.3 or lower, (2) a minor allele frequency (MAF) lower than 0.05
(3) out of Hardy-Weinberg equilibrium $(p<1 \times 10-6)$, (4) a call rate lower than $95 \%$, or (5) a Gprobs score lower than 0.90. Exactly 5,815,690 SNPs passed the QC process.

\section{Data cleaning and analysis}

We conducted analyses using PLINK [14], a whole genome association analysis toolset. We excluded SNPs that exceeded thresholds for Hardy-Weinberg Equilibrium $[15,16]$ (-hwe 0.00001), missing genotype rate (-geno $0.05)$, and minor allele frequency (-maf 0.01 ) on the Knight ADRC and ADNI datasets. Then, we excluded individuals with a missing genotype rate greater than $2 \%$ (-mind 0.02).

With the cleaned data, we conducted a linear regression for all remaining SNPs, within each data set, to test for an association with prolactin levels, adjusting for age, gender, and the first two principle components generated using EigenSoft [17, 18]. We then performed a meta-analysis across ADNI and Knight ADRC for CSF and another meta-analysis across ADNI and Knight ADRC for plasma, each accounting for sample size, $p$-values, and direction of effect using the default METAL [19] settings.

We retained all SNPs that had a meta-analysis p-value less than $5 \times 10^{-8}$ and that had the same direction of effect in both the Knight ADRC and ADNI datasets, in both resulting meta-analysis files. We then looked for SNPs that were replicated in both the significant CSF and plasma meta-analysis resulting files. We searched for these SNPs in the NHGRI catalog of published genome-wide association studies [20]. (downloaded October 12th, 2015) for known disease associations. We then used RegulomeDB [21] and functional annotations

Table 1 Significant SNPs were scattered across chromosomes 2, 6, 7, 8 and 17, with the majority of the SNPs being on chromosome 6. These 12 SNPs were all significant in both the blood plasma and CSF. Information on the SNPs includes chromosome, reference and alternate allele, minor allele frequency, predicted function, the gene the SNP is found in or near, RegulomeDB score, and the meta-analysis $p$-values for plasma and CSF

\begin{tabular}{|c|c|c|c|c|c|c|c|c|c|c|}
\hline \multirow[t]{2}{*}{ SNP } & \multirow[t]{2}{*}{ Chr } & \multirow{2}{*}{$\begin{array}{l}\text { Base Pair } \\
\text { Position }\end{array}$} & \multirow{2}{*}{$\begin{array}{l}\text { Major } \\
\text { Allele }\end{array}$} & \multirow{2}{*}{$\begin{array}{l}\text { Minor } \\
\text { Allele }\end{array}$} & \multirow[t]{2}{*}{ MAF } & \multirow{2}{*}{$\begin{array}{l}\text { Predicted } \\
\text { Function }\end{array}$} & \multirow[t]{2}{*}{ Gene } & \multirow{2}{*}{$\begin{array}{l}\text { RegulomeDB } \\
\text { score }\end{array}$} & \multicolumn{2}{|c|}{ Meta-analysis $p$-value } \\
\hline & & & & & & & & & Plasma & CSF \\
\hline rs12548348 & 8 & 70430077 & A & G & 0.1222 & Intronic & SULF1 & No Data & $6.288 \mathrm{e}-11$ & $9.841 e-26$ \\
\hline rs13408093 & 2 & 62251682 & A & T & 0.0699 & Intronic & TRIB2 & 5 & $6.881 \mathrm{e}-10$ & $2.119 e-25$ \\
\hline rs1150703 & 6 & 28184260 & G & T & 0.0919 & ncRNA_exonic & TOB2P1 & 5 & $3.276 \mathrm{e}-09$ & $7.011 \mathrm{e}-26$ \\
\hline rs988083 & 6 & 28177588 & C & T & 0.1220 & Intergenic & ZNF192P1,TOB2P1 & 5 & $3.276 \mathrm{e}-09$ & $7.011 e-26$ \\
\hline rs988084 & 6 & 28177492 & C & T & 0.1218 & Intergenic & ZNF192P1,TOB2P1 & 6 & $3.276 \mathrm{e}-09$ & $7.011 \mathrm{e}-26$ \\
\hline rs73726888 & 7 & 15021811 & $\mathrm{~T}$ & $C$ & 0.0893 & UTR3 & GIMAP7 & 6 & $4.209 \mathrm{e}-09$ & $6.438 \mathrm{e}-24$ \\
\hline rs8073041 & 17 & 47498253 & $\mathrm{~T}$ & A & 0.0731 & Intergenic & PHB,LOC101927207 & 6 & 7.87e-09 & $2.169 \mathrm{e}-10$ \\
\hline rs1150701 & 6 & 28183886 & A & C & 0.1410 & ncRNA_exonic & TOB2P1 & 6 & $1.184 \mathrm{e}-08$ & $1.443 e-26$ \\
\hline rs1150702 & 6 & 28184097 & $A$ & T & 0.1410 & ncRNA_exonic & TOB2P1 & 5 & $1.184 \mathrm{e}-08$ & $1.443 \mathrm{e}-26$ \\
\hline rs1233712 & 6 & 28193131 & G & A & 0.1406 & UTR5 & ZSCAN9 & 4 & $1.184 \mathrm{e}-08$ & $1.443 e-26$ \\
\hline rs79268972 & 17 & 47531241 & $\mathrm{~T}$ & G & 0.0749 & Intergenic & PHB,LOC101927207 & 5 & $2.547 \mathrm{e}-08$ & $8.169 \mathrm{e}-12$ \\
\hline rs77482998 & 7 & 47532356 & T & C & 0.0665 & Intronic & TNS3 & 5 & $4.608 \mathrm{e}-08$ & $7.983 e-12$ \\
\hline
\end{tabular}


from wAnnovar $[22,23]$ to identify SNPs that are biologically likely to modify gene function or expression. RegulomeDB scores range from " $1 \mathrm{a}$ " to " 6 ". Lower scores indicate stronger evidence that the SNP affects gene regulation based on both empirical data, such as ChIPseq, and whether the SNP is within a known transcription factor binding motif. We generated regional association plots using SNAP [24] for regions of interest and explored

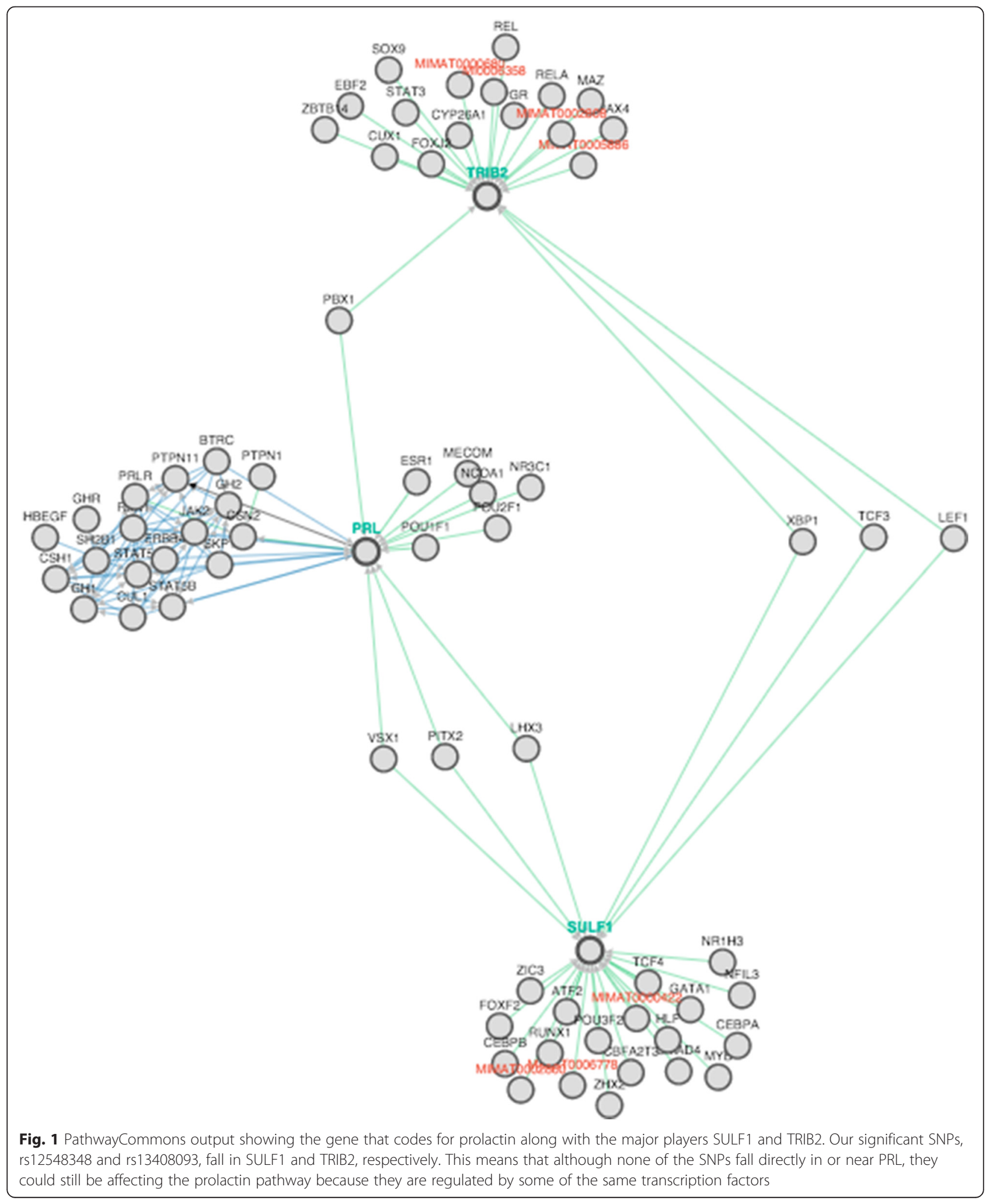


whether any genes of interest are part of the same pathway or regulatory network using PathwayCommons [25]. For SNPs where linkage disequilibrium data is unknown in SNAP, we modified the SNAP source code to plot all SNPs in the region regardless of linkage disequilibrium status and omit $\mathrm{r}^{2}$ values. By default, SNAP only plots SNPs with a known $r^{2}$ greater than 0 . We also generated $\mathrm{q}-\mathrm{q}$ plots in $\mathrm{R}$ to check for evidence of inflation of $p$-values.

\section{Results}

We identified 37 SNPs associated with prolactin levels in plasma and 666 SNPs associated with prolactin levels in CSF (Additional files 1 and 2), none of which are located in or around the $P R L$ gene. Significant SNPs were spread across 21 chromosomes for the CSF results and across 10 different chromosomes for the plasma results. There are several hits on chromosome 6 , but all are more than 5 million base pairs away from where the $P R L$ gene is located. There were 12 SNPs in common between the plasma and CSF results (Table 1), 6 of which were on chromosome 6 , approximately 6 million base pairs away from the $P R L$ gene. RegulomeDB scores for the 12 SNPs ranged from 4 to 6 and MAFs ranged from 0.06 to 0.14 . None of the 12 SNPs were found in the NHGRI catalog of published genome-wide association studies. The q-q plots demonstrated no evidence of inflation (genomic inflation factor $=1.0$; Additional files 3 and 4). According to PathwayCommons, PRL, SULF1, and TRIB2 are all regulated by some of the same transcription factors (Fig. 1) including PBX1, XBP1, TCF3, LEF1, VSX1, $P I T X 2$, and $L H X 3$. There were no other known relationships among the genes identified in this study.

\section{Discussion}

Twelve SNPs were significantly associated with prolactin levels in both plasma and CSF, 6 are located on chromosome 6 and the remaining 6 SNPs are scattered across chromosomes 2, 7, 8, and 17. The 6 SNPs on chromosome 6 cluster in and around ZSCAN9, TOB2P1, and ZNF192P1, according to Annovar, though visualizing the SNPs' locations in the NCBI viewer shows that 3 of the 6 SNPs fall within a ZSCAN9 intron for one specific transcript (XM_011514877.1) as well as within TOB2P1-a pseudogene that falls within the same intronic region of

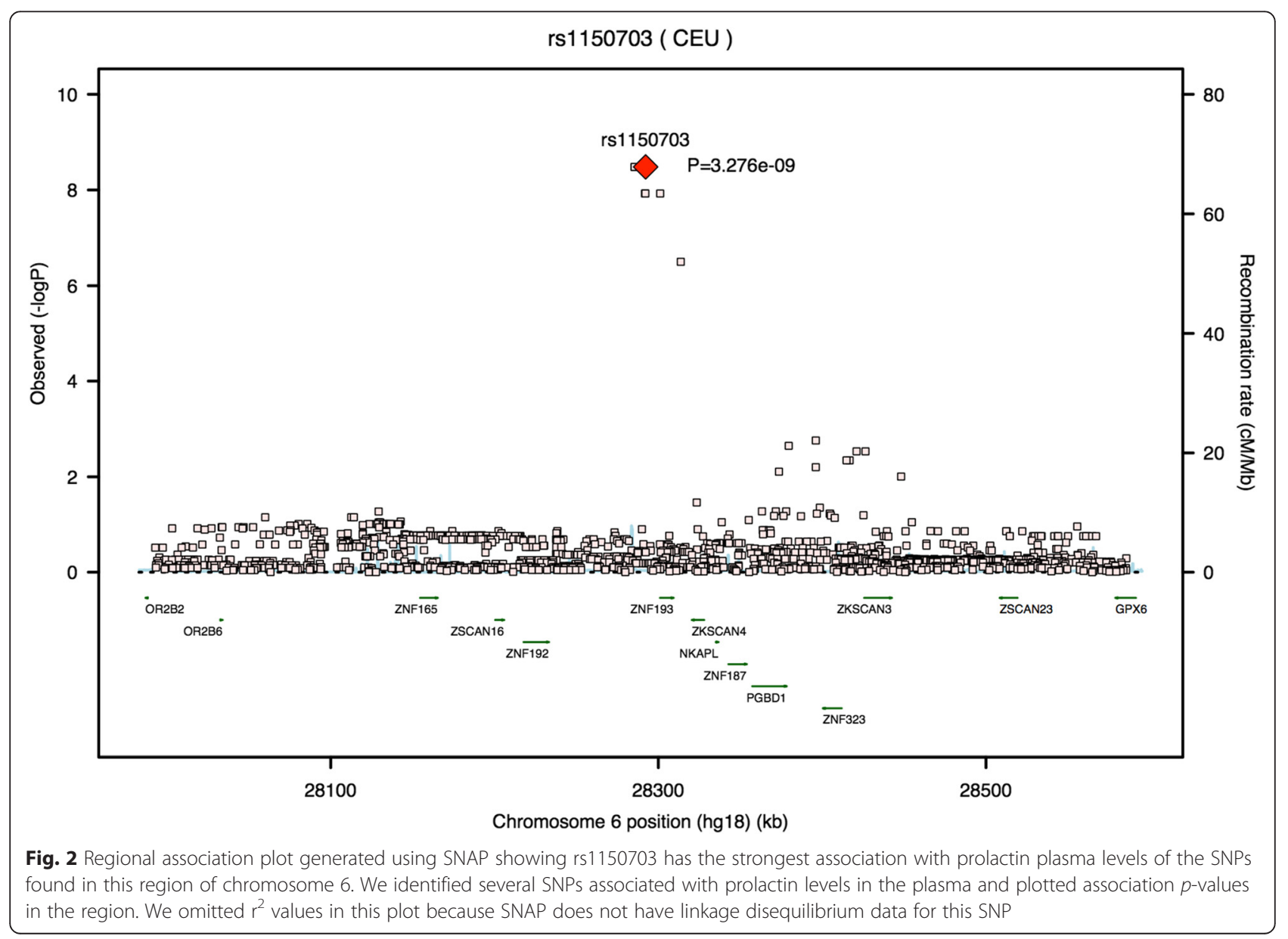


ZSCAN9. SNP rs1233712 is in the $5^{\prime} \mathrm{UTR}$ region of ZSCAN9. SNPs rs988083 and rs988084 are between ZNF192P1 and TOB2P1, according to Annovar. ZNF192P1 is also a pseudogene that is proximal to ZSCAN8. In short, all 6 SNPs on chromosome 6 are located in or around ZSCAN8 and ZSCAN9, both of which are protein-coding genes, while 3 of the 6 fall directly within a pseudogene (TOB2P1). Of the significant SNPs on chromosome 6, rs1150703 was most significantly correlated with prolactin levels in plasma (Fig. 2) while rs1150701 was most significantly correlated with prolactin levels in CSF (Fig. 3).

The remaining 6 SNPs are located on chromosomes 2, 7,8 , and 17, where 2 of the SNPs are intergenic, 3 are intronic, and one is located in a $3^{\prime}$ UTR region (Table 1). SNP rs12548348 is an intronic SNP within the SULF1 gene on chromosome 8 and was most significantly associated with prolactin levels in plasma out of the 12 found in common between the two fluids. It was also one of most significantly associated with prolactin levels in CSF. SNPs rs13408093 and rs77482998 are intronic SNPs within the TRIB2 (chromosome 2) and TNS3 (chromosome 7) genes, respectively. SNPs rs8073041 and rs79268972 are intergenic SNPs that are both located on chromosome 17 between the gene $P H B$ and a non-coding RNA LOC101927207. The next closest protein-coding gene is NGFR. SNP rs73726888 is located in the 3'UTR region of GIMAP7 on chromosome 7. While rs77482998 (TNS3) and rs73726888 (GIMAP7) are both located on chromosome 7 , they are distant from each other on opposite arms of the chromosome, suggesting their associations with prolactin levels are independent of each other.

While there is no direct evidence that any of these markers directly impact prolactin expression, it appears that PRL, SULF1, and TRIB2 in that they are all regulated by common transcription factors, including $P B X 1$, $X B P 1, T C F 3, L E F 1, V S X 1, P I T X 2$, and $L H X 3$. It is possible that these genes and variants are involved in $P R L$ regulation through more complex biological relationships. This may be significant because genes regulated by the same transcription factor are often active in the same tissues at the same time $[26,27]$.

\section{Conclusions}

In summary, we have identified significant and replicable association between several genetic variants in both

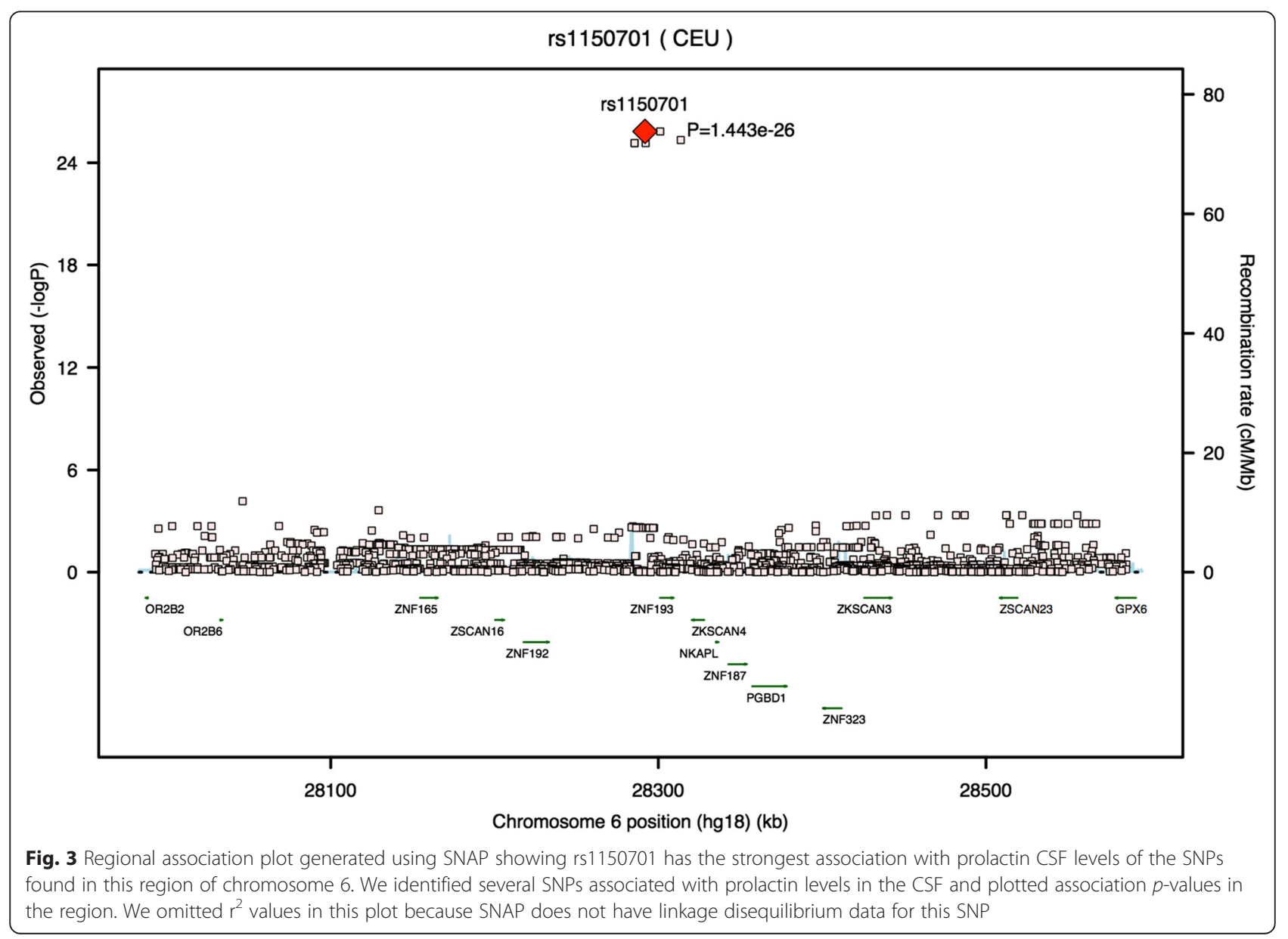


plasma and CSF levels of prolactin. These results provide a foundation for a better understanding of prolactin regulation, and in turn the host of phenotypes in which prolactin plays a role, including lactation, immunoregulation, apoptosis and T-lymphocyte function [1-3, 7]. Future work on these associated markers will provide meaningful insights into these phenotypes.

\section{Additional files}

Additional file 1: File contains a table of SNPs significantly associated with prolactin levels in blood plasma by meta-analysis. (DOCX $130 \mathrm{~kb}$ )

Additional file 2: File contains a table of SNPs significantly associated with prolactin levels in CSF by meta-analysis. (DOCX 183 kb)

Additional file 3: File contains a $\mathrm{Q}-\mathrm{Q}$ plot of the plasma data used in this study. (DOCX $74 \mathrm{~kb}$ )

Additional file 4: File contains a Q-Q plot of the CSF data used in this study. (DOCX $76 \mathrm{~kb}$ )

\section{Abbreviations}

PIF, prolactin inhibitory factors; ADNI, Alzheimer's Disease Neuroimaging Initiative: CSF, Cerebrospinal Fluid; eOTL, expression quantitative trait locus; Knight ADRC, Knight-Alzheimer's Disease Research Center at Washington University School of Medicine; SNP, single nucleotide polymorphism; UTRs, untranslated regions

\section{Acknowledgements}

The authors acknowledge that many scientists contributed in developing the clinical and genetic resources necessary to collect these data and complete this project. The authors also gratefully acknowledge the efforts of hundreds of individuals who participated as subjects in these studies.

The NIH (R01 AG035053, R01 AG042611, P50 AG05681, P01 AG03991, P01 AG026276), the Alzheimer's Association (MNIRG-11-205368), and the Brigham Young University Gerontology Program provided support for this work. We also acknowledge the Alzheimer's Disease Genetics Consortium (ADGC) and Genetic and Environmental Risk for Alzheimer's Disease Consortium (GERAD) for providing genotype data used in this work. GERAD was supported by the Medical Research Council (Grant nu 503480), Alzheimer's Research UK (Grant nu 503176), the Wellcome Trust (Grant nu 082604/2/07/Z) and German Federal Ministry of Education and Research (BMBF): Competence Network Dementia (CND) grant nu 01GI0102, 01GI0711, 01GI0420. CHARGE was partly supported by the NIH/NIA grant R01 AG033193 and the NIA AG081220 and AGES contract N01-AG-12100, the NHLBI grant R01 HL105756, the Icelandic Heart Association, and the Erasmus Medical Center and Erasmus University. ADGC was supported by the NIH/NIA grants: U01 AG032984, U24 AG021886, U01 AG016976, and the Alzheimer's Association grant ADGC-10-196728. Data collection and sharing for this project was funded by the Alzheimer's Disease Neuroimaging Initiative (ADNI) (National Institutes of Health Grant U01 AG024904) and DOD ADNI (Department of Defense award number W81XWH-12-2-0012). ADNI is funded by the National Institute on Aging, the National Institute of Biomedical Imaging and Bioengineering, and through generous contributions from the following: Alzheimer's Association; Alzheimer's Drug Discovery Foundation; BioClinica, Inc.; Biogen Idec Inc.; Bristol-Myers Squibb Company; Eisai Inc.; ElanPharmaceuticals, Inc.; Eli Lilly and Company; F. Hoffmann-La Roche Ltd and its affiliated company Genentech, Inc.; GE Healthcare; Innogenetics, N.V.; IXICO Ltd.; Janssen Alzheimer Immunotherapy Research \& Development, LLC.; Johnson \& Johnson Pharmaceutical Research \& Development LLC.; Medpace, Inc:; Merck \& Co., Inc.; Meso Scale Diagnostics, LLC. NeuroRx Research; Novartis Pharmaceuticals Corporation; Pfizer Inc.; Piramal Imaging; Servier; Synarc Inc.; and Takeda Pharmaceutical Company. The Canadian Institutes of Health Research is providing funds to support ADNI clinical sites in Canada. Private sector contributions are facilitated by the Foundation for the National Institutes of Health (www.fnih.org). The grantee organization is the Northern California Institute for Research and Education, and the study is coordinated by the Alzheimer's Disease Cooperative Study at the University of California, San Diego. ADNI data are disseminated by the Laboratory for Neuro
Imaging at the University of Southern California. Some of the samples used in this study were genotyped by the ADGC and GERAD. ADGC is supported by grants from the NIH (\#U01AG032984) and GERAD from the Wellcome Trust (GR082604MA) and the Medical Research Council (G0300429).

Portions of data used in preparation of this article were obtained from the Alzheimer's Disease Neuroimaging Initiative (ADNI) database (adni.loni.usc.edu). As such, the investigators within the ADNI contributed to the design and implementation of ADNI and/or provided data but did not participate in analysis or writing of this report.

The ADNI Executive Committee consists of: Michael Weiner, MD UC San Francisco; Paul Aisen, MD UC San Diego; Ronald Petersen, MD, PhD Mayo Clinic, Rochester; Clifford R. Jack, Jr., MD Mayo Clinic, Rochester; William Jagust, MD UC Berkeley; John Q. Trojanowki, MD, PhD U Pennsylvania; Arthur W. Toga, PhD USC; Laurel Beckett, PhD UC Davis; Robert C. Green, MD, MPH Brigham and Women's Hospital/Harvard Medical School; Andrew J. Saykin, PsyD Indiana University; John Morris, MD Washington University St. Louis; Leslie M. Shaw University of Pennsylvania. A complete listing of ADNI investigators can be found at: http://adni.loni.usc.edu/wp-content/uploads/ how_to_apply/ADNI_Acknowledgement_List.pdf

\section{Declarations}

Publication of this article was funded by Brigham Young University's Department of Biology.

This article has been published as part of BMC Genomics Volume 17 Supplement 3, 2016: Selected articles from the 12th Annual Biotechnology and Bioinformatics Symposium: genomics. The full contents of the supplement are available online at https://bmcgenomics.biomedcentral.com/ articles/supplements/volume-17-supplement-3.

\section{Availability of data and materials}

Data are available to researchers by applying to the respective organizations, ADNI and ADGC consortia. Application is required to protect participant confidentiality. The ADNI data are available at (http://adni.loni.usc.edu/), the Knight ADRC data are available through dbGAP (http://www.ncbi.nlm.nih.gov/gap).

\section{Authors' contributions}

LS, SP, MB and JSKK carried out data analysis. LS and ME annotated and analyzed the SNPs for significance and drafted the manuscript. All other authors participated in the conception of the project and obtaining data. All authors read and approved the final manuscript.

\section{Competing interests}

The authors declare that they have no competing interests.

Ethics approval and consent to participate

Data was obtained and analyzed under approval of the Brigham Young University Institutional Review Board.

\section{Consent for publication}

Not applicable.

\section{Author details}

'Department of Biology, Brigham Young University, Provo, UT 84602, USA. ${ }^{2}$ Biology and Biomedical Sciences, Washington University, St. Louis, MO 63110 , USA. ${ }^{3}$ Department of Neuroscience Icahn School of Medicine, New York, NY 10029, USA.

Published: 29 June 2016

\section{References}

1. Freeman ME, Kanyicska B, Lerant A, Nagy G. Prolactin: structure, function, and regulation of secretion. Physiol Rev. 2000;80:1523-631.

2. Ben-Jonathan $N$, LaPensee $C R$, LaPensee $E W$. What can we learn from rodents about prolactin in humans? Endocr Rev. 2008;29:1-41.

3. Grattan DR. 60 years of neuroendocrinology: the hypothalamo-prolactin axis. J Endocrinol. 2015;226:T101-22.

4. Peter Fitzgerald TGD. Prolactin and dopamine: what is the connection? A review article. J Psychopharmacol Oxf Engl. 2008;22:12-9.

5. Larsen CM, Grattan DR. Prolactin, neurogenesis, and maternal behaviors. Brain Behav Immun. 2012;26:201-9. 
6. Melmed S, Casanueva FF, Hoffman AR, Kleinberg DL, Montori VM, Schlechte $\mathrm{JA}$, et al. Diagnosis and treatment of hyperprolactinemia: an Endocrine Society clinical practice guideline. J Clin Endocrinol Metab. 2011;96:273-88.

7. Nithya Krishnan OT. Prolactin suppresses glucocorticoid-induced thymocyte apoptosis in vivo. Endocrinology. 2003;144:2102-10.

8. Aluise CD, Sowell RA, Butterfield DA. Peptides and proteins in plasma and cerebrospinal fluid as biomarkers for the prediction, diagnosis, and monitoring of therapeutic efficacy of Alzheimer's disease. Biochim Biophys Acta. 2008;1782:549-58.

9. John SK, Kauwe MHB. Genome-wide association study of CSF levels of 59 Alzheimer's disease candidate proteins: significant associations with proteins involved in amyloid processing and inflammation. PLoS Genet. 2014;10, e1004758.

10. Cruchaga C, Kauwe JSK, Harari O, Jin SC, Cai Y, Karch CM, et al. GWAS of cerebrospinal fluid tau levels identifies risk variants for Alzheimer's disease. Neuron. 2013;78:256-68.

11. Anne M, Fagan MAM. Inverse relation between in vivo amyloid imaging load and CSF Aß42 in humans. Ann Neurol. 2006;59:512-9.

12. Trojanowski JQ, Vandeerstichele H, Korecka M, Clark CM, Aisen PS, Petersen $\mathrm{RC}$, et al. Update on the biomarker core of the Alzheimer's Disease Neuroimaging Initiative subjects. Alzheimers Dement J Alzheimers Assoc. 2010;6:230-8.

13. Kauwe JSK, Bailey MH, Ridge PG, Perry R, Wadsworth ME, Hoyt KL, et al. Genome-wide association study of CSF levels of 59 Alzheimer's disease candidate proteins: significant associations with proteins involved in amyloid processing and inflammation. PLoS Genet. 2014;10, e1004758.

14. Purcell S, Neale B, Todd-Brown K, Thomas L, Ferreira MAR, Bender D, et al. PLINK: a tool set for whole-genome association and population-based linkage analyses. Am J Hum Genet. 2007;81:559-75.

15. Wigginton JE, Cutler DJ, Abecasis GR. A note on exact tests of hardy-Weinberg equilibrium. Am J Hum Genet. 2005;76:887-93.

16. Jan Graffelman VM. The mid p-value in exact tests for Hardy-Weinberg equilibrium. Stat Appl Genet Mol Biol. 2013;12:433-48.

17. Patterson N, Price AL, Reich D. Population structure and eigenanalysis. PLoS Genet. 2006;2, e190

18. Price AL, Patterson NJ, Plenge RM, Weinblatt ME, Shadick NA, Reich D. Principal components analysis corrects for stratification in genome-wide association studies. Nat Genet. 2006;38:904-9.

19. Willer CJ, Li Y, Abecasis GR. METAL: fast and efficient meta-analysis of genomewide association scans. Bioinforma Oxf Engl. 2010;26:2190-1.

20. Welter D, MacArthur J, Morales J, Burdett T, Hall P, Junkins H, et al. The NHGRI GWAS Catalog, a curated resource of SNP-trait associations. Nucleic Acids Res. 2014;42:D1001-6.

21. Boyle AP, Hong EL, Hariharan M, Cheng Y, Schaub MA, Kasowski M, et al. Annotation of functional variation in personal genomes using RegulomeDB. Genome Res. 2012;22:1790-7.

22. Wang K, Li M, Hakonarson H. ANNOVAR: functional annotation of genetic variants from high-throughput sequencing data. Nucleic Acids Res. 2010;38, e164.

23. Chang X, Wang K. wANNOVAR: annotating genetic variants for personal genomes via the web. J Med Genet. 2012;49:433-6.

24. Johnson AD, Handsaker RE, Pulit SL, Nizzari MM, O'Donnell CJ, de Bakker PIW. SNAP: a web-based tool for identification and annotation of proxy SNPs using HapMap. Bioinformatics. 2008;24:2938-9.

25. Cerami EG, Gross BE, Demir E, Rodchenkov I, Babur Ö, Anwar N, et al. Pathway Commons, a web resource for biological pathway data. Nucleic Acids Res. 2011;39:D685-90.

26. Visel A, Minovitsky S, Dubchak I, Pennacchio LA. VISTA Enhancer Browser-a database of tissue-specific human enhancers. Nucleic Acids Res. 2007:35: D88-92.

27. Kleftogiannis D, Kalnis P, Bajic VB. DEEP: a general computational framework for predicting enhancers. Nucl Acids Res. 2015;43:e6-e6.

\section{Submit your next manuscript to BioMed Central and we will help you at every step:}

- We accept pre-submission inquiries

- Our selector tool helps you to find the most relevant journal

- We provide round the clock customer support

- Convenient online submission

- Thorough peer review

- Inclusion in PubMed and all major indexing services

- Maximum visibility for your research

Submit your manuscript at www.biomedcentral.com/submit
Biomed Central 